\title{
Effects of vibration and passive resting on muscle stiffness and restitution after submaximal exercise analyzed by elastography
}

\author{
WIESŁAW ChWAŁA ${ }^{1 *}$, PAWEŁ POGWIZD ${ }^{2}$ \\ ${ }^{1}$ Department of Biomechanics, University of Physical Education in Krakow, Kraków, Poland. \\ ${ }^{2}$ Research and Development Department of Vitberg, Nowy Sącz, Poland.
}

\begin{abstract}
Purpose: The research aimed to assess the influence of vibration therapy on acceleration of muscles regeneration process after physical effort. Verification of the pace of motor capabilities recovery in fatigued muscles was performed with the use of the measurement method applying shear-wave elastography. It took into account an alteration in muscle stiffness affected by created passive stresses and accompanying deformations connected with muscles work above their resting length. Methods: The research included 42 young males aged 20-24. They all were a homogeneous group regarding body build indices (body height $175.4 \pm 8.1 \mathrm{~cm} ;$ body mass $75.5 \pm 8.32 \mathrm{~kg}$ ) and the level of physical activity. Muscles of lower limbs (hip and knee joints extensors and flexors plantaris) were subject to submaximal exercise of the auxotonic muscle work character after a $90 \mathrm{~s}$ warm-up. The exercise comprised 20 -fold overcoming the load at the level of $75 \%$ of maximal strength abilities without rest, by alternating between extension and flexion of lower limbs joints at the leg press placed at $45^{\circ}$ angle upward and monitoring of angle setting of lower limbs joints. The post-exercise restitution used a vibration stimulus of variable frequency ranging from 20 to $50 \mathrm{~Hz}$ and amplitude of $0.5 \mathrm{~mm}$, and passive resting in the control group. For an objective assessment of the applied vibration effect, a 5-fold measurement of rectus femoris muscles stiffness of both limbs at the middle of venter took place after each stage of the experiment. Results: After warm-up, a considerable increase of the examined muscles stiffness, that did not vary significantly, was observed. In the DW experimental group (vibration massage), where post-exercise restitution was enhanced by vibration, significantly lower values of muscle stiffness were registered after each of the post-exercise regeneration stages in relation to the DB control group (passive rest). After the I regeneration stage, a value of the $\Delta \sigma_{-}$rl-s variable $(p<0.05)$ lower by $16 \%$ was noticed in favour of the group enhanced by vibration. Whereas, values of muscles stiffness after the II stage of regeneration, $\Delta \sigma \_$r2-s were by $14 \%$ lower in favour of DW group $(p<0.005)$. Conclusions: Enhancement of the post-exercise relaxation of muscles by vibration treatment significantly lowered the values of post-exercise muscles stiffness and turned out to be a more effective method of restitution than passive resting. Recovery of the level of muscles stiffness from before the exercise was much slower in the control group than in the one enhanced by vibration. The improved method of muscle stiffness measurement with the use of shear-wave elastography with a special head attachment turned out to be a useful tool to assess post-exercise muscles restitution.
\end{abstract}

Key words: elastography, vibration, biomechanics, muscle stresses, regeneration, physical effort

\section{Introduction}

Intensive physical activity leads to fatigue resulting in worsening of exercise capacity demonstrated by lowered intensity and efficiency of muscles work. In the case of maximal physical effort, the feeling of fatigue occurs already in the first seconds [25]. So far, many differentiated and objective methods of assessing the fatigue levels have been characterised [11].

Fatigue is a common phenomenon in sport. It subsides after properly long rest, frequently enhanced by biological regeneration of the organism. Different techniques and methods are used to increase efficiency of the post-exercise restitution process. One of the criteria for assessment of the applied resting method is ex-

\footnotetext{
* Corresponding author: Wiesław Chwała, Department of Biomechanics, Institute of Biomedical Sciences, University of Physical Education in Krakow, Al. Jana Pawła II 78, 31-571 Kraków, Poland. Tel. 504303896, e-mail: wieslaw.chwala@awf.krakow.pl

Received: December 21st, 2020

Accepted for publication: February 23rd, 2021
} 
amining whether it causes faster organism recovery than passive rest [4].

Currently, the most commonly applied forms of biological regeneration comprise of sauna, cryotherapy, steam baths, hydro massage, pearl bath, whirlpool bath and massage. Especially massage has gained recognition in sport. Many leading athletes from different disciplines believe that this type of treatment supports organism after training effort and competitions.

Most of the applied methods of body biological regeneration concerns effects after a finished training. In the view of each training unit efficiency, the key issue is restoration of motor capabilities after subsequent exercise series that can shorten breaks between the series while simultaneously maintaining ability to develop high motor efficiency of the organism in subsequent repetition of applied training exercises.

The way the mechanical vibrations (WBV) applied in the present study affect the organism is close to a classical massage using vibration techniques. Vibration causes an increase of blood vessels volume, which facilitates blood flow in processes removing harmful substances and delivering oxygen to fatigued tissues [7]. Oscillations of surface tissue make the deeper tissues oscillate as well, thus accelerating metabolism. Vibration can facilitate absorption of any substances in tissues and secretion of substances from tissues to blood. It has a stimulating effect on secretory activity of all glands and causes an increase of muscle tissue, its elasticity and strength [3].

Vibration treatment can be applied to a single muscle, a muscles group and even the whole body. Recent studies suggest that vibrations can limit the phenomenon of delayed muscles soreness. Lau et al. [18] showed that a 30-minute-long vibration treatment after training was effective in suppressing DOMS (delayed onset muscle soreness) and restoring motion range of elbow joint flexors after eccentric muscles exercises. Some researchers who applied vibration training after physical effort claim that it positively affected muscles regeneration [23]. However, other authors [3], [10] suggest that application of vibrations within $24 \mathrm{hrs}$ after exercise can cause additional loss of strength and inhibit the effect of biological regeneration in this period. The above-mentioned tests results indicate divergence of individual authors' views concerning vibration therapy dedicated at biological regeneration of muscles that previously became fatigued. A significant issue affecting efficiency of vibrations can be the character and outline of the vibration treatment. Another essential matter seems to be search for an accurate, reliable and objective meas- urement tool enabling verification of researchers' current views on effectiveness of vibration treatments in the process of post-exercise body biological regeneration.

It appears necessary to apply new measurements methods in researches to enable objective confirmation of applied techniques of biological regeneration efficiency.

Due to dual (active and passive) character of muscle response generated during eccentric work, the use of sEMG may not be sufficient. The ultrasound elastography, that uses the phenomenon of suppressing shearwave by tissues, might become a promising method to verify current reports. Recently, there have been publications where authors applied elastography [6], [13] to assess biomechanical properties of muscle tissue.

Mechanical properties of the tissue can be described examining its deformation under the influence of an acting force [2]. Soft tissues get deformed under the influence of an external force. It is described by the Young's modulus $(E)$ which expresses a typical for the assessed biological material stress dependence $(\sigma)$ on relative deformation $(\varepsilon): E=\sigma / \varepsilon$, within flexible deformations described by the Hooke's law.

Shear-wave elastography relies on deformation of tissue under the influence of remotely created force of acoustic effect and is a quantitative image of local alterations in tissue stiffness. Tissue deformations induce a shear wave spreading perpendicularly to the direction the subsequent acoustic impulses are produced in. The velocity of the shear wave is determined by correlation of subsequent images. After the velocity is defined, a shear modulus and then Young's modulus are determined. The shear wave induced by the acoustic wave depends on viscoelastic properties of biological tissue [2]. As post-exercises and post-injury changes in muscles and tendons are connected with their biomechanical functions impairment, a search has been conducted since elastography was introduced in order to find clinical applications of the method to forecast risks of tissue injuries in athletes and to assess efficiency of physiotherapy or training both in a healthy and affected by a pathological process musculotendinous tissue.

The aim of the research was to compare the efficiency of two forms of resting (vibration effect and passive rest) within the scope of enhancing muscles regeneration after single submaximal effort. Another aim was to determine the influence of particular stages of pre-exercise regeneration on recovery of muscles stiffness to the optimal level obtained after warm-up. 


\section{Materials and methods}

The tests included 42 males aged 20-24 of medium body height $175.4 \pm 8.1 \mathrm{~cm}$, body mass $75.5 \pm 8.32 \mathrm{~kg}$, who had not experienced orthopaedic injuries in the previous year or any other illnesses that could significantly affect the obtained measurements results.

Qualified persons participated in equal weekly physical activity connected with realization of the university curriculum and did not practise sport professionally. Moreover, they declared that in the last week since the beginning of tests they did not change considerably their lifestyle, dietary habits or undertake physical activity that could significantly influence measurements results that were taken during the tests. Before the measurements, the course of tests was precisely explained to participants and they delivered informed consent to take part in them. The Bioethical Commission approved the tests $(\mathrm{KB} / 245 / \mathrm{FI})$.

The group of 42 persons was randomly divided into 2 groups of 21 people. The participants qualified to the DW group (kinetic exercise - vibration massage) underwent vibration massage following the original protocol described below. Whereas, solely passive rest was applied after physical effort for the participants in the DB control group (kinetic exercise - passive rest). Each group was tested by the same researchers with the use of the same measurement tools and following the same measurement procedures. The beginning of the experiment was preceded by a 10-minute rest in a relaxing position lying on the back on the examination coach. Then, in resting conditions (lying on the back) an initial measurement of muscle stress condition with the use of shear-wave elastography was performed. The examination followed the standards of the Polish Ultrasound Society [15].

Each participant started their activity with a standard warm-up consisting of alternate flexions and extensions in lower limbs joints on the leg press in a sitting position with the trunk set on the press back at $45^{\circ}$ angle to the ground. The load value for all the examined was $200 \mathrm{~N}$. Each of the participants had to do the load value 45 times within ca. 90 seconds maintaining individual pace of subsequent cycles of the warm-up. Positions of sections in joints were monitored by a goniometer. In the starting position the lower limb was extended and the foot was in a neutral position (right angle between the foot and shin). During flexion in knee joints, the angle between the calf and thigh measured at internal side was $80^{\circ}\left(0^{\circ}\right.$ - full extension; $80^{\circ}$ maximal - flexion). The angle in hip joint was determined by flexion angles in knee joints and feet as well as individual dimensions of lower limbs sections. Constant angle setting in knee and ankle joints was enabled by adjustable construction of the leg press. Pelvis and the rest of the trunk were stabilised using lock belts.

Another measurement of muscle stiffness was taken after finished warm-up following all methodological determinants as in the first trial.

In order to assess the maximal level of strength abilities of lower limbs muscles generated altogether by extensors of hip and knee joints and flexors plantaris, an initial measurement of maximal strength in isometric contraction was taken after warm-up, lying on the back at a measuring stand with the press set at $45^{\circ}$ angle upward and settings of lower limbs joints as given above.

In subsequent stage each of the participants was examined with submaximal physical effort particularly overloading muscle groups of lower limbs aiming at their fatigue. The effort comprised 20-times overcoming the load at the level of $75 \%$ of maximal strength abilities without rests, by alternate flexion and extension of lower limbs joints on the leg press in a sitting position (trunk set at $45^{\circ}$ angle to the ground). Immediately after the trial, the muscle stress condition was assessed for the third time.

After the exercise, each participant took part in the first stage of rest. The regeneration time for each of the group was $20 \mathrm{~min}$. The DW group members underwent vibration treatment in a position relaxing for lower limbs joints muscles. Oscillations were directed locally to extensors of hip and knee joints and flexor plantaris of both lower limbs. The carrier of vibration mattress was a dedicated armchair that enabled taking a set resting position. Whereas participants of the control group rested passively in the same position and in identical armchairs as DW persons.

Another (IV) stage of muscle stress measurement took place after 20 minutes of vibration treatment for DW group and passive rest for DB group.

The last $(\mathrm{V})$ stage of assessing muscle stress in the examined muscle groups took place after subsequent 20 minutes of passive rest in both groups.

\subsection{Vibration effect}

Vibration massage of the muscles engaged in the effort was conducted during rest after submaximal exercise of a kinetic character. The experiment employed a vibration therapy device made by Vitberg. An original vibration programme was worked out for the purpose of the research and it generated differenti- 
ated breaks in vibration stimulus effect lasting from 1 to 4 seconds during the whole 20-min-long treatment. The outline of vibration effect was directed at proper reactivity of muscle fibres [8]. The device generated oscillations of frequency ranged $20-50 \mathrm{~Hz}$, of amplitude $<0.5 \mathrm{~mm}$. The tests involved the use of vibration mattress with an optional vibration module of total application surface allowing for simultaneous (without changing the position of the mattress and person) treatment for muscle groups of both lower limbs.

\subsection{Measurement of muscle tissue stress}

In order to assess results of the applied post-exercise regeneration, the selected muscles were examined with the use of shear-wave elastography. The tests were performed five times: before warm-up, before and after kinetic exercise, and after vibration exercise and passive rest. It was done with the Supersonic Aixplorer device with linear head SL18-5 (5-18 MHz) equipped with the function of elastographic assessment. The device uses the ultrasound system with the U1traFastTM platform that enables obtaining images 200 times faster than conventional ultrasound systems. Shear-wave elastography allows for measurement of tissues and blood flows in real time in reproducible way. The Q-BocTM pack included automatic tools calculating the required values of variables. The values of tissues stiffness were displayed on the screen both in units of waves propagation basic velocities $\left[\mathrm{ms}^{-1}\right]$, and also of pressure values $[\mathrm{kPa}]$ reflecting the tissue stiffness of the measurement area. The device head was

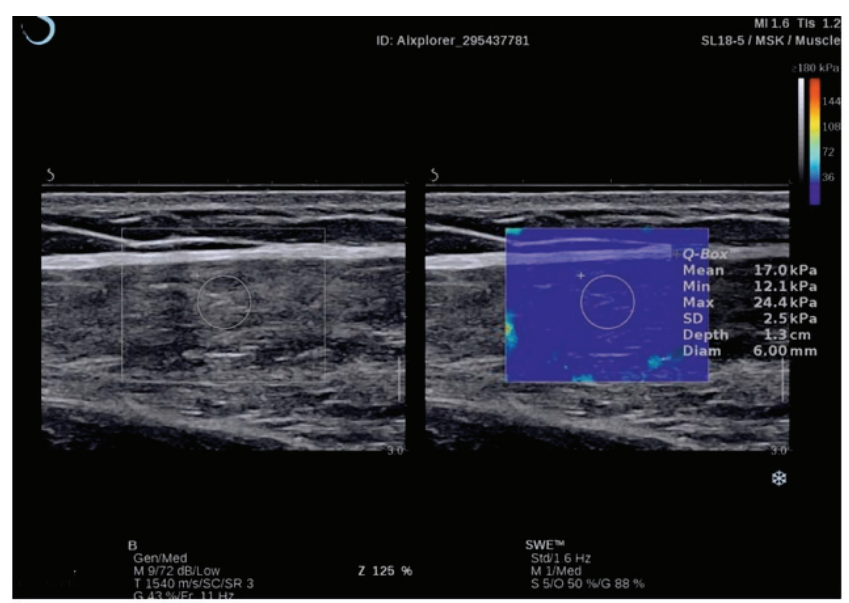

Fig. 1. Presentation of elastogram on the ultrasound device monitor. On the left - a visible greyscale image; on the right - a visible image in which the elastogram of the examined tissues is presented in colour scale placed at the middle of venter of rectus femoris. The obtained elastograms used a range of colours to present the condition of muscle tissue stress.

The ultrasound scanner head was set perpendicularly towards the assessed tissues, always in the same place marked at the middle of rectus femoris venter in right and left lower limb. The size of the measurement area was selected so that it was located inside the rectus femoris venter. In the conducted tests, the measurement area was a circle of $6 \mathrm{~mm}$ diameter, whereas the measurement was taken at $13 \mathrm{~mm}$ depth from the skin surface. Within the measurement area, the researchers avoided large blood vessels, bone and tendon structures. During the tests, the original attachment designed and 3D-printed with a profiled hole for the head applied for measurements was used. It enabled the researchers to avoid measurement errors connected with qualifications of the head operator. Differentiated pressure of the head onto skin was a serious drawback of the elaborations published previously [6], [13]. The space between the head and muscle venter was filled up with gel. No additional pressure of the head onto skin at the examination spot was applied. The pressure always equalled the weight of the head itself and amounted to $3 \mathrm{~N}$. All the above-listed factors caused reproducibility of the head setting during each measurement as well as maximal objectivization of the obtained results of soft tissues stiffness. The obtained ultrasound images of the examined areas of muscles were registered in the ultrasound scanner memory. For each measurement stage, 3 images of each limb were registered to read stress values from them, and next the mean value of muscle stress in both lower limbs was calculated. The value was then compared to the results of muscle stiffness obtained identically in subsequent measurement stages.

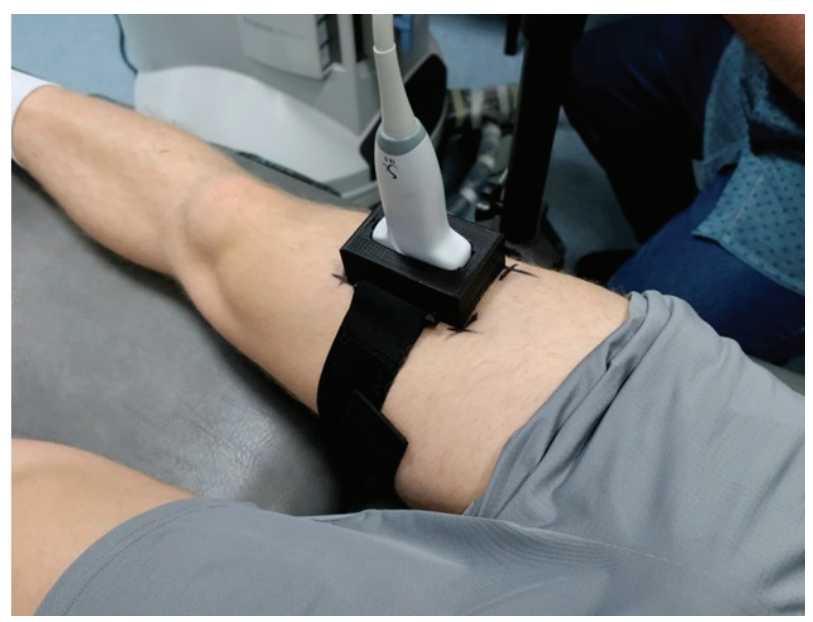

Fig. 2. Measurement of muscle stress with the use of an attachment to hold the USG scanner head - right lower limb 
The results were statistically analysed with the use of the STATISTICA 13.3 PL software pack. The recorded changes in muscle stress values influenced by warm-up, exercise and both regeneration stages were used to determine growth of absolute values of mean muscle stiffness in relation to the initial values (measured before warm-up) in subsequent stages of the experiment. The significance of statistical differences between mean values of growths in muscle stresses recorded in subsequent stages was examined with the Friedman's Anova test for reproducible measurement with the Dunn's post-hoc test. Assessment of differences significance between mean values of analysed variables growths in both groups was performed with the use of one-way analysis of variance (ANOVA) applying the Tukey's test. In case of lack of normal distribution or lack of variance homogeneity tested with Levene's test, the Anova Kruksal-Wallis and Dunn's post-hoc test were applied.

\section{Results}

In Figure 3, percentage values of changes in muscles stiffness between subsequent stages in relation to the initial value for each group taken as $100 \%$ are shown. In Figures 4 and 5, the obtained results of the Anova analysis for reproducible measurements in all the examined groups are presented.

After warm-up, a significant and similar increase of stiffness was observed in both groups. The results of statistical analyses indicate that in each group the mean growths of muscle stiffness $\Delta \sigma_{-} \mathrm{w}$-s, caused by physical effort were statistically significantly higher

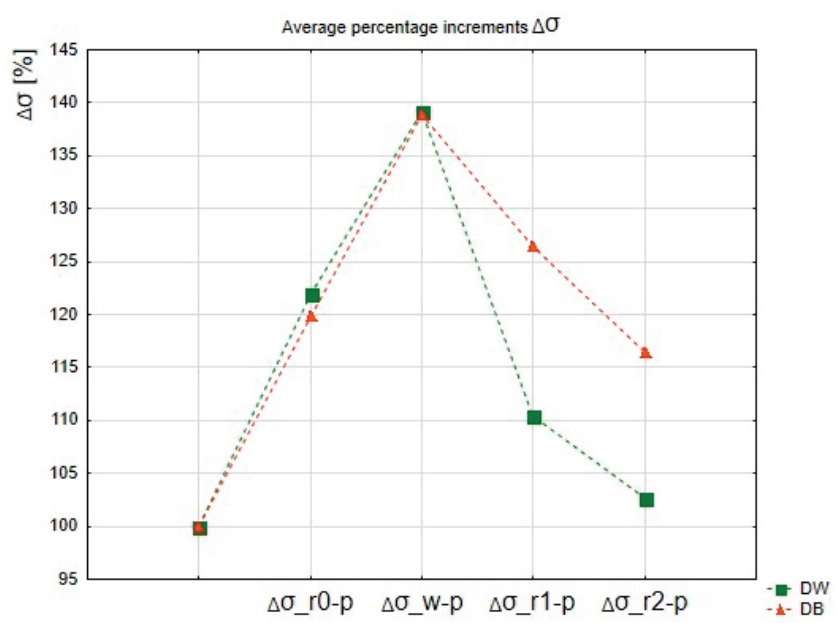

Fig. 3. Percentage changes of muscle stiffness in relation to initial stiffness in subsequent stages of the experiment in experimental (DW) and control (DB) groups

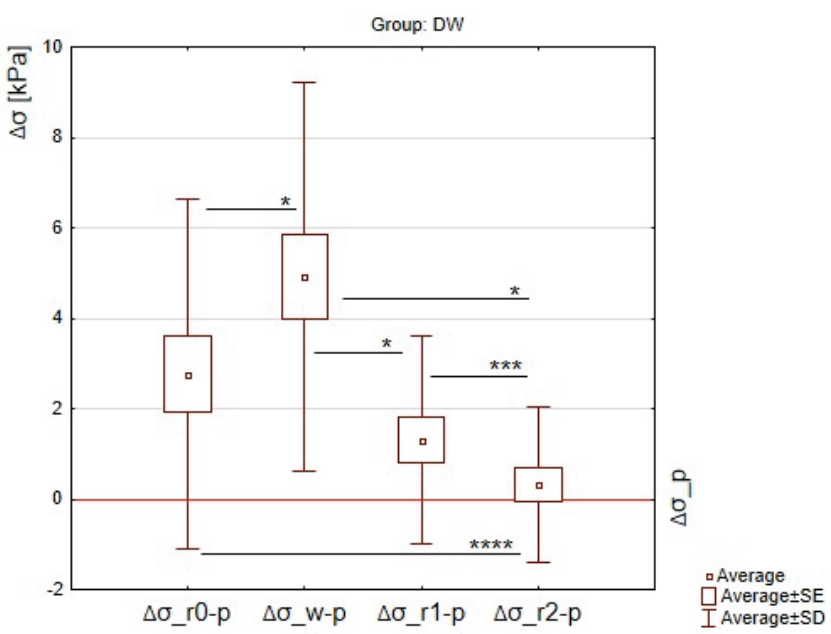

Fig. 4. Graphic presentation of the Friedman's ANOVA analysis results for values of muscle stiffness changes compared to initial stiffness in subsequent stages of the experiment for the experimental group (DW)

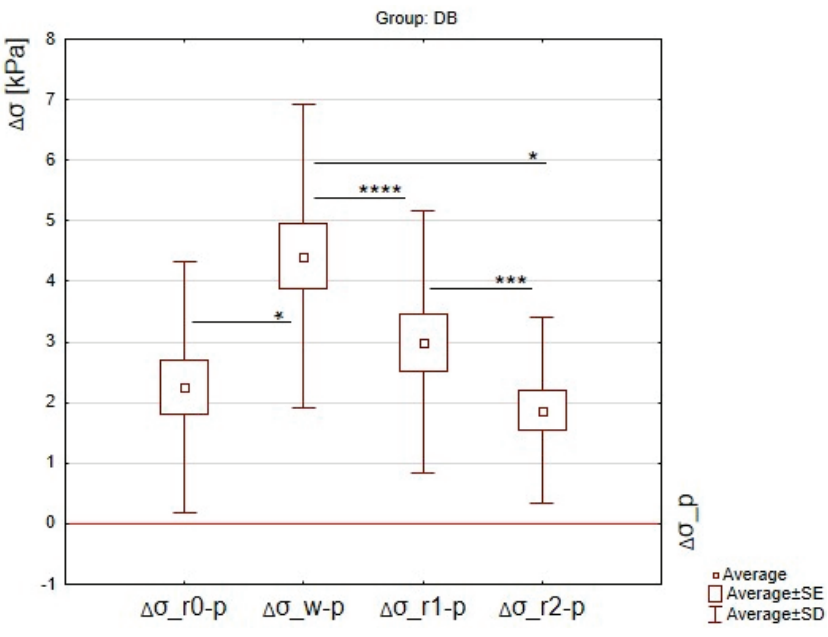

Fig. 5. Graphic presentation of the Friedman's ANOVA analysis results for values of muscle stiffness changes compared to initial stiffness in subsequent stages of the experiment for the control group (DB)

compared to mean growths $\Delta \sigma$ r0-s registered after warm-up, $(p<0.001)$. For the pair of variables $\left(\Delta \sigma_{-}\right.$w-s vs $\Delta \sigma_{-} \mathrm{r} 0$-s) in the DW group, a growth in muscle stress stiffness was recorded at the level of $2.1 \mathrm{kPa}$ (Fig. 4), which meant about $14 \%$ growth in relation to the values of muscle stress observed directly after warm-up. In the DB control group the growth of muscle stiffness was almost identical at $2.2 \mathrm{kPa}$ (Fig. 5) for analogous pair of variables which was about $16 \%$ change. In both DW $(p<0.05)$ (Fig. 4) and DB $(p<0.001)$ (Fig. 5), the registered changes in muscle stiffness values $\Delta \sigma \mathrm{r} 1$-s after the first regeneration stage reached significantly lower values than after exercise $\Delta \sigma_{-}$rw-s, which indicates that I stage of 
Table 1. Mean values of muscle stiffness changes in relation to initial stiffness in subsequent stages in experimental (DW) and control (DB) groups

\begin{tabular}{|c|c|c|c|c|}
\hline & $\begin{array}{c}\Delta \sigma_{-} \mathrm{r} 0-\mathrm{s}[\mathrm{kPa}] \\
\bar{x} \pm \mathrm{s}\end{array}$ & $\begin{array}{c}\Delta \sigma_{-} \mathrm{w}-\mathrm{s}[\mathrm{kPa}] \\
\bar{x} \pm \mathrm{s}\end{array}$ & $\begin{array}{c}\Delta \sigma_{-} \mathrm{rl}-\mathrm{s}[\mathrm{kPa}] \\
\bar{x} \pm \mathrm{s}\end{array}$ & $\begin{array}{c}\Delta \sigma_{-} \mathrm{r} 2-\mathrm{s}[\mathrm{kPa}] \\
\bar{x} \pm \mathrm{s}\end{array}$ \\
\hline DW & $2.8 \pm 3.87$ & $4.9 \pm 4.30$ & $1.3 \pm 2.29$ & $0,3 \pm 1,72$ \\
\hline DB & $2.3 \pm 2.07$ & $4.4 \pm 2.50$ & $3.0 \pm 2.16$ & $1,9 \pm 1,53$ \\
\hline$\Delta \sigma[\mathrm{kPa}]$ & 0.5 & 0.5 & 1.7 & 1.6 \\
\hline$p$ & NS & NS & $<0.05$ & $<0.005$ \\
\hline
\end{tabular}

$p$ - the level of statistical significance of differences between mean values of muscle stiffness in the examined groups. NS - statistically insignificant.

restitution only partially lowered muscles stiffness in relation to the initial state. However, a more visible effect of lowering muscle stiffness was observed in experimental group than in the control one (Fig. 4). Rest with vibration stimulation of muscles caused lowering stiffness below the level recorded after warm-up, completely eliminating the influence of post-exercise fatigue, whereas, when analysing values of changes after warm-up $\Delta \sigma \_$r0-s and the second regeneration stage $\Delta \sigma$ r2-s, statistically significant differences were observed only for the DW group $(p<0.05)$. The second stage of passive regeneration lowered muscle stiffness in the DW group to the level close to the idle value while in the control group - to the after warmup level (Fig. 4).

The result of Friedman's Anova test for reproducible measurements indicated occurrence of statistically significant differences between values of muscle stiffness growths after exercise $\Delta \sigma_{-} \mathrm{w}-\mathrm{s}$, and each of the regeneration stages $\left(\Delta \sigma_{-} \mathrm{r} 1-\mathrm{s}, \Delta \sigma_{-} \mathrm{r} 2-\mathrm{s}\right)$ at different level of probability in both groups. For the DW group, the registered lowering of muscle stiffness subsequently after the first and second rest periods was respectively $21 \%$ and $26 \%$, which made a decrease of the observed stiffness by $3.6 \mathrm{kPa}$ and $4.6 \mathrm{kPa}$, respectively, compared to the value $\Delta \sigma_{-} \mathrm{w}-\mathrm{s}$ recorded after physical effort. In the case of the DB control group, a remarkably slower pace of muscles stress values decrease subsequently after each regeneration stage was noticed. The recorded falls were about $9 \%$ and $16 \%$, respectively, which corresponded to changes by $1.4 \mathrm{kPa}$ and $2.5 \mathrm{kPa}$. Presence of significantly lower values of stiffness decreases $(p<0.01)$ was found in both groups for recorded values of muscle stiffness assessing changes that occurred after the second regeneration in relation to the values registered after the first regeneration stage $\left(\Delta \sigma_{-} \mathrm{r} 1\right.$-s vs $\Delta \sigma_{-} \mathrm{r} 2$-s). In the DW group, the recorded decrease between I and II regeneration stages was $1 \mathrm{kPa}$, which made a change by about $7 \%$, whereas for the $\mathrm{DB}$ group the values were $1.1 \mathrm{kPa}$ and $8 \%$, respectively (Fig. 4).
In Table 1, results of testing significance of differences between mean values of muscle stress in both examined groups are presented.

The results of the one-way analysis of variance confirmed the occurrence of statistically significant differences for mean decreases of muscle stiffness recorded after the first regeneration $\Delta \sigma_{-} \mathrm{rl}$-s between the groups $(p<0.05)$. For the DW group, a decrease of muscle stiffness $\Delta \sigma$ r1-s, after I regeneration stage compared to the initial value reached the value of $1.7 \mathrm{kPa}$ which made about $16 \%$ fall and was significantly higher than in the DB group. Considerable differences in muscle stiffness were also maintained in both groups after the second regeneration stage. Mean stiffness values after II regeneration stage $\Delta \sigma_{-} \mathrm{r} 2$-s in the DW group were lower by about $14 \%(1.6 \mathrm{kPa})$ compared to the DB group $(p<0.005)$.

\section{Discussion}

Estimated engagement of muscles during exercise and efficient elimination of fatigue symptoms remain one of the main challenges in biomechanics. Classically, the level of muscles activity during exercise is often assessed with the use of the surface electromyography (sEMG). However, there are known limitations connected with applying the method while assessing muscle strength during kinetic exercises [11]. Due to changes of muscles length during kinetic exercise, different muscle strengths can be produced for the same levels of the muscle bioelectrical activation if it works at different lengths. The method does not provide any information on the passive strength whose participation in total stress value becomes very important after exceeding resting length (eccentric work). Moreover, occurrence of neuromuscular fatigue as well as auxotonic form of work practically make it impossible to determine connection between sEMG amplitude and the generated strength [20]. 
There are scientific reports claiming that, in the case of fatigue resulting from physical effort, the relaxation should have an active form. Mika et al. [21] proved that mild exercises applied after intensive physical effort can accelerate elimination of muscle fatigue symptoms and are more efficient than passive rest. According to Lattier et al. [16], a very important factor influencing acceleration of post-exercise relaxation and ability to undertake another effort is elimination of lactate from blood and muscles. Cafarelli et al. [7] believe that the processes can be accelerated by vibration which causes increase of blood circulation and may have the key importance in eliminating harmful substances from organism and delivering oxygen to muscles. The authors of the reports rarely observed differences between the influence of active and passive rest on parameters obtained during subsequent effort [17]. Moreover, Barnes et al. [3] think that additional work realised by muscles due to vibration effect could cause losses at the level of strength abilities of the examined muscles. The theory is supported by researches whose authors suggest that an increase of muscles contractions is accompanied by a decrease of their strength [9]. Other authors report that WBV does not help in muscles regeneration after exercise because when a muscle is damaged, it alters efficiency of WBV as a corrective measure [10]. Divergences of the obtained results can be caused by a different protocol of test exercise and measurement methods assessing efficiency of the applied method of relaxation. Another factor that has a significant influence on post-exercise regeneration effect can be improperly selected parameters of the vibration stimulus, especially frequency, amplitude, duration of stimulation and breaks, and position in which the vibration effect is applied.

According to Weber et al. [24], the best indication of proper muscles regeneration after physical effort is recovery of the muscles to the initial level or reaching hyper compensation.

The most important information resulting from the tests is the observation that enhancing post-exercise muscle relaxation by vibration treatment affects lowering muscle stiffness more efficiently than passive rest. Vibration treatment on groups of lower limbs muscles fatigued due to physical effort that was applied during the first regeneration stage positively influenced their stiffness recovery close to the level recorded after warm-up. After the first regeneration stage, even slightly lower level of the engaged muscles stiffness was registered than before the physical effort. This observation suggests that it is possible to shorten duration of vibration treatment to achieve the most optimal effect.
After warm-up, the muscles are well prepared for physical effort featuring slightly higher stiffness values than in resting conditions [5]. It ensures effective undertaking subsequent efforts in a training unit. However, stiffness cannot be too high because of unfavourable phenomenon of increasing muscles viscosity.

In the control group, a 20-minute rest did not allow obtaining pre-exercise level of muscle stiffness, which suggests that muscles did not recover completely and were not ready to undertake subsequent submaximal effort. Slower pace in decrease of muscle stress values after exercise in the control group indicates that passive rest cannot affect relaxation of muscle tissue as efficiently as vibration treatment, which significantly lowers effectiveness of a training due to necessary longer resting breaks. It is also confirmed by tests of Mathesoni et al. [19] who found out that vibration stimulation more beneficially affects relaxation and lowering of muscle stress than resting in a lying position. Also, Issurin et al. [14] demonstrated in their research that vibration can cause relaxation of stressed muscles. Such tests results are confirmed by Opara et al. [22], who think that vibration massage ensures muscles relaxation and its general effect can also rely on relaxation and lowering emotional tensions. WBV induces slight muscles contractions based on muscles lengthening and shortening. Therefore, muscles with applied vibration treatment are forced to realise additional work [3]. That is why muscles regeneration, apart from essential restoration of energy substrates, should aim at fast recovery of stiffness values close to the values obtained after warm-up.

The second stage of passive rest that was applied in both groups was characterised by lower pace of muscle restitution than in the case of vibration therapy and resulted in recovery of muscle stiffness close to the resting level in the experimental group, whereas in the control group - to the level obtained after warm-up.

Recapitulating both stages of post-exercise restitution, it should be stated that recovery of muscle stiffness after submaximal effort to the level obtained after warm-up required about two times shorter vibration effect (ca. $15 \mathrm{~min}$ ) than passive rest (ca. $30 \mathrm{~min}$ ).

The level of regaining motor abilities of fatigued muscles is frequently assessed by examining motor effect (measurement of strength abilities, force in countermovement jump, etc.). However, these are indirect measurements and their results can be affected by other factors, not included in the measurement. Elastography offers direct measurement of stiffness in selected areas of muscle venter with the use of the ultrasound shear wave suppression level. The Supersonic 
Aixplorer device used in the tests that measure muscle stiffness after post-exercise fatigue by shear-wave elastography can be an alternative for current methods of assessing restitution of muscle strength abilities. Bouillard et al. [6] demonstrated a strong linear dependence between the value of stiffness and angular momentum of the examined muscle structure while applying in their researches a measurement of muscles with the use elastography to estimate alterations in muscle strength abilities. According to them, the measurement of muscle stiffness can be a more precise method of measuring muscle strength abilities than examining functional biopotentials sEMG. Also Alfuraih et al. [1] suggest that shear-wave elastography ensures precise and reliable measurement of muscle stiffness, however, it should be emphasized that, as for all ultrasound measurements, it depends on the operator (mainly on the pressure of the head onto tissues found below) introducing the element of human error. That is why the authors of the present study applied an original attachment to hold the USG scanner head in which it allowed to minimize the level of measurement errors and to obtain reproducible results.

\section{Conclusions}

1. Application of vibration effect significantly more efficiently lowered the level of post-exercise muscle stiffness than passive rest.

2. No differentiation between the experimental and control groups was found within the range of increasing resting stiffness of muscles after warm-up and auxotonic submaximal effort.

3. Application of vibration effect during active muscle restitution between exercises can significantly shorten breaks in a training unit and improve efficiency of the training.

4. As a result of implemented improvements of the research method, application of shear-wave elastography turned out to be a useful and reliable tool for assessment of the muscle stiffness level at different stages of the experiment.

\section{Acknowledgements}

This research was founded within the framework of the program of the Minister of Science and Higher Education (Poland) under the name 'Regional Initiative for Perfection' within the years 2019-2022, project No. 022/RID/2018/19.

\section{References}

[1] Alfuraih A.M., O’Connor P., Tan A.L., Hensor E.M., LADAS A., EMERY P., WAKEFIELD R.J., Muscle shear wave elastography in idiopathic inflammatory myopathies: a case-control study with MRI correlation, Skeletal Radiology, 2019, 48 (8), 1209-1219.

[2] Bamber J., Cosgrove D., Dietrich C.F., Fromageau J., Bojunga J.., Caliada F, CANTisani V. et al., EFSUMB guidelines and recommendations on the clinical use of ultrasound elastography. Part 1: Basic principles and technology, Ultraschall Med., 2013, 34 (2), 169-184.

[3] Barnes M.J., Perry B.G., Mundel T., Cochrane D.J., The effects of vibration therapy on muscle force loss following eccentrically induced muscle damage, Eur. J. Appl. Physiol., 2012, 112 (3), 1189-1194.

[4] BARNeTT A., Using Recovery Modalities between Training Sessions in Elite Athletes. Does it help?, Sports Medicine, 2006, 36 (9), 781-796.

[5] BERDEJo DEL FreSNo D., Competitive warm-up in basketball: literature review and proposal, Revista de Ciencias del Depporte, 2011, 7 (2), 101-116.

[6] Bouillard K., Nordez A., Hug F., Estimation of individual muscle force using elastography, PLoS One, 2011, 6 (12), 1-7.

[7] Cafarelli E., Sini J., Carolan B., Liebesinan J., Vibratory Massage and Short-Term Recovery from Muscular Fatigue, Int. J. Sports Med., 1990, 11 (6), 474-478.

[8] Cardinale M., Lim J., The acute effects of two different whole body vibration frequencies on vertical jump performance, Med. Sport, 2003, 56 (4), 287-292.

[9] Chapman D.W., Newton M., McGuigan M., Nosaka K., Effect of lengthening contraction velocity on muscle damage of the elbow flexors, Med. Sci. Sports Exerc., 2008, 40 (5), 926-933.

[10] DabBs N.C., Brown L.E., GARnER J.C., The Effects of whole body vibration on vertical jump performance following exercise induced muscle damage, International Journal of Kinesiology and Sports Science, 2014, 2 (1), 23-30.

[11] DANEK J., Wojtasik W., SzUlC A., Measurement of maximal isometric torque and muscle fatigue index of the knee muscles in male athletes, Acta Bioeng. Biomech., 2019, 21 (3), 31-37.

[12] Hug F., Can muscle coordination be precisely studied by surface electromyography?, J. Electromyogr. Kinesiol., 2011, 21 (1), 1-12.

[13] Hug F., Tucker K., Gennisson J.L., TANTER M., NordeZ A., Elastography for Muscle Biomechanics: Toward the Estimation of Individual Muscle Force, Exerc. Sport Sci. Rev., 2015, 43 (3), 125-133.

[14] Issurin V.B., Liebermann D.G., Tenenbaum G., Effect of vibratory stimulation on maximal force and flexibility, J. Sports Sci., 1994, 12 (6), 561-566.

[15] JAKubowski W., Standards of ultrasound examinations, Roztocze School of Ultrasound, 2011 (in Polish).

[16] Lattier G., Millet G.Y., Martin A., Martin V., Fatigue and Recovery after High-Intensity Exercise. Part II: Recovery Interventions, Int. J. Sports Med., 2004, 25 (7), 509-515.

[17] LaU S., Berg K., Lattin R.W., NoBle J., Comparison of active and passive recovery of blood lactate and subsequent performance of repeated work bouts in ice hockey players, J. Strength Cond. Res., 2001, 15 (3), 367-371.

[18] LAU W.Y., NOSAKA K., Effect of vibration treatment on symptoms associated with eccentric exercise surface induced 
muscle damage, Am. J. Phys. Med. Rehabil., 2011, 90 (8), 648-657.

[19] Matheson D.W., Edelson R., Hiatrides D., Newkirk J., Twinem K., Thurston S., Relaxation Measured by EMG as a Function of Vibrotactile Stimulation, Biofeedback and Self-Regulation, 1976, 1 (3), 285-292.

[20] McCullough M.B., Domire Z.J., Reed A.M., Amin S., YTTERBERG S.R., CHEN Q. et al., Evaluation of muscles affected by myositis using magnetic resonance elastography, Muscle Nerve, 2011, 43 (4), 585-590.

[21] Mika A., Oleksy Ł., Mikolajczyk E., MarchewKa A., Evaluation of the effectiveness of various methods supporting postexercise muscle relaxation using electromyography surface ( $s E M G)$, Medical Journal of the Rzeszow University and the National Medicines Institute, Warsaw, 2011, 1, 25-39 (in Polish).
[22] Opara J.A., Mehlich K., GieremeK K., Szwejkowski W., Vibratory massage for complementing training in sports and physiotherapy, Physiotherapy, 2010, 18 (1), 61-66 (in Polish).

[23] Padulo J., Di Giminiani R., Ibba G., Zarrouk N., Moalla W., Attene G., Migliaccio G.M., Pizzolato F., Bishop D., CHAMARI K., The acute effect of whole body vibration on repeated shuttle-running in young soccer players, Int. J. Sports Med., 2014, 35 (1), 49-54.

[24] Warren G.L., Lowe D.A., Armstrong R.B., Measurement tools used in the study of eccentric contraction-induced injury, Sports Med., 1999, 27 (1), 43-59.

[25] ŻoŁĄDŹ J.A., What determines the strength, power and endurance of human skeletal muscles?, StatSoft Poland, 2003 (in Polish). 